\title{
Theory of Quantum Optical Control of Single Spin in a Quantum Dot
}

\author{
Pochung Chen, ${ }^{1,2}$ C. Piermarocchi,${ }^{1,3}$ L. J. Sham, ${ }^{1}$ D. Gammon, ${ }^{4}$ and D. G. Steel ${ }^{5}$ \\ ${ }^{1}$ Department of Physics, University of California San Diego, La Jolla, California 92093-0319 \\ ${ }^{2}$ Department of Chemistry, University of California Berkeley, Berkeley, California 94720-1640 \\ ${ }^{3}$ Department of Physics and Astronomy, Michigan State University, East Lansing, Michigan 48824-2320 \\ ${ }^{4}$ Naval Research Laboratory, Washington, D.C. 20375-5347 \\ ${ }^{5}$ Harrison M. Randall Laboratory of Physics, The University of Michigan, Ann Arbor, MI 48109-1120
}

(Dated: November 1, 2018)

\begin{abstract}
We present a theory of quantum optical control of an electron spin in a single semiconductor quantum dot via spin-flip Raman transitions. We show how an arbitrary spin rotation may be achieved by virtual excitation of discrete or continuum trion states. The basic physics issues of the appropriate adiabatic optical pulses in a static magnetic field to perform the single qubit operation are addressed.
\end{abstract}

\section{INTRODUCTION}

Spin-flip Raman spectroscopy has been widely applied to the study of the properties of donors and acceptors

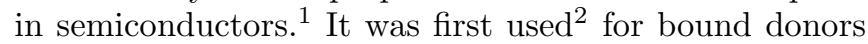
in $\mathrm{CdS}$, and coherent phenomena such as Raman spinecho were subsequently observed 3.4 Coherent spectroscopic techniques have attracted new interest due to their potential utilization in the control and manipulation of simple quantum mechanical systems. In particular, the application of coherent Raman processes to qubit operations in quantum information processing has been suggested for a variety of systems, for example, an electron spin in a semiconductor quantum dot,,$\frac{5}{5}$ trapped ions, 6.7 molecules, $\frac{8}{\underline{e}}$ and rare-earth impurities in crystals,$\frac{9}{.}$. The optical rotation of electron spins has been demonstrated in semiconductor quantum wells ${ }^{10.11}$

In this paper we show how spin-flip Raman optical transitions can lead to the full quantum control of a single electron spin in a semiconductor quantum dot. This involves optically connecting the two electron spin ground states to trions as the intermediate excited states. A trion is a bound state of an exciton with the electron in the dot. The role of using one or more discrete states in the dot and continuum states in the host are analyzed. The constraints in the design of the optical pulses to preserve the adiabaticity necessary for a high fidelity of the control are discussed. There are two possible advantages of optical control compared with other control schemes, in the femtosecond time scale of theultrafast laser pulses and the efficiency and flexibility of pulse shaping techniques ${ }^{12,13}$ for quantum operations.

The extant experimental situation provides a sound foundation towards implementation of our theory. A semiconductor quantum dot charged with one electron presents a strong analogy to a single bound donor. However, the spin-flip Raman experiments in semiconductors mentioned above involve ensemble measurements whereas quantum control would require experiment on a single dot. The quantum control of a single exciton in a single dot by coherent optical techniques is affirmed by the experimental demonstration of the Rabi oscillations ${ }^{14.15 .16}$ Magneto-luminescence of trion levels from a single dot reported recently ${ }^{20}$ forms the basis toward quantum control.

The spin of an electron in a quantum dot has been proposed as a qubit for the implementation of quantum computers $\stackrel{21}{2}$ It has the advantage of an extremely long

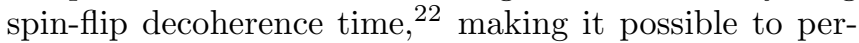
form a large number of quantum operations. We provide here a full theory for the quantum control of single qubit operations using optical pulses. When combined with the proposal to couple spins in neighboring quantum dots by optically induced RKKY interaction, 23 one has a complete scheme to build a scalabe quantum computer based on spins in quantum dots via optical control. Although in principle the optically controlled RKKY interaction alone is sufficient for universal computation, 24 the requirement of at least three physical qubits to form a single qubit makes the route of using a complete set of single qubit operations plus a two-qubit conditional operation perhaps less difficult for the purpose of a minimalist physical demonstration of two-qubit "computation". The idea of using Raman schemes to realize single qubit operation was mentioned by Imamoglu et $a l^{\underline{\underline{5}}}$ and Pazy et $a l^{25}$. Here we expand this suggestion, providing a full theory of single spin rotation by means of optical pulses with an explicit formulation.

The paper is organized as follows. In section II we discuss the selection rules and the effects of an external static magnetic field in optical transitions involving trions in quantum dots (QD). While we focus on QDs generated by mono-layer fluctuations in narrow quantum wells 26 as an example, the theory is applicable to other kinds of dots, such as self-assembled dots. We introduce one particular configuration of light polarization and magnetic field orientation that realizes a lambda system. Section III explains how to perform adiabatic Raman transitions in this lambda system via one trion state. The link between the parameters of the optical pulses and the angle and the axis of the spin rotation is given in the most general case. The dependence of the spin rotation on the orientation of the magnetic field is presented. The suppression of decoherence in the adiabatic regime is shown by a numerical solution of the dissipative dy- 
namics based on the Liouville equation and explained by a qualitative discussion. Section $[\nabla$ examines the effects of the adiabatic Raman transitions via multiple discrete or continuum trion states. Section V summarizes the key results.

\section{TRION STATES IN A CHARGED DOT}

We consider a system of electrons and holes confined in a quantum dot described by the Hamiltonian

$$
H^{e h}=H_{0}+H_{\text {Coul }}+H_{B}^{e}+H_{B}^{h}+H_{C}(t),
$$

where $H_{0}$ represents the part of the non-interacting electron and hole states and $H_{C o u l}$ the Coulomb interaction between them. The effects of the external magnetic field on the electrons and holes are given by

$$
\begin{aligned}
H_{B}^{e} & =\frac{1}{2} \mu_{B} \sum_{n j \alpha \beta} g_{j}^{e} e_{n \alpha}^{\dagger} \sigma_{\alpha \beta}^{j} e_{n \beta} \\
\text { and } \quad H_{B}^{h} & =\frac{1}{2} \mu_{B} \sum_{m j \alpha \beta} g_{j}^{h} B_{j} h_{m \alpha}^{\dagger} \sigma_{\alpha \beta}^{j} h_{m \beta},
\end{aligned}
$$

where $\hbar$ is set to unity, $\sigma_{\alpha \beta}^{j}$ denotes the $\alpha \beta$-th element of the Pauli matrix in the Cartesian direction $j(=x, y, z)$, and $e_{n \alpha}\left(h_{n \alpha}\right)$ represents the annihilation operator of an electron (hole) in the dot at the $n$-th level and spin (pseduospin) $\sigma$ up or down. Note that the hole levels include the doubly degenerate heavy and light hole states. Although in some III-V compounds such as GaAs the electron $g$-tensor, $g^{e}$, is approximately isotropic $\stackrel{20}{=}$ we allow here for the anisotropic case with the principal axes along the Cartesian axes with $z$ being in the growth direction of the semiconductor heterostructure. In the dipole and rotating wave approximation the light-matter interaction is

$$
H_{C}(t)=\sum_{i \sigma} \Omega_{i \sigma}(t) e^{-i \omega_{\sigma} t} e_{i \sigma}^{\dagger} h_{i \sigma}^{\dagger}+h . c .
$$

where $\Omega_{i \sigma}$ denotes a time-dependent complex Rabi frequency following the envelope of the optical pulse centered at the frequency $\omega_{\sigma}$, propagating in the growth direction with circular polarization $\sigma$ (left-handed $\sigma=-1$ and right-handed $\sigma=+1$ ). For simplicity, in the heavyhole exciton associated with polarization $\sigma$, the conduction electron spin component is taken to be dominated by $e_{n,-\sigma}(\operatorname{spin}-\sigma 1 / 2)$ and the valence hole $h_{m \sigma}$ (spin $\sigma 3 / 2$ ). For the $\sigma$ light-hole exciton, the components are $e_{n, \sigma}$ and $h_{m \sigma}$ (spin $\sigma 1 / 2$ ). Correction of this simplification is straightforward in computation 27 but will unnecessarily complicate the exposition of the optical processes below. The interaction $H_{C}(t)$ represents the control Hamiltonian to be designed for the manipulation of the spins. The semiclassical approximation is appropriate since the intensity of the laser field involved is strong enough to render the photon fluctuation effects negligible. The combined effects of the spin-orbit interaction and the dot confinement depress the light hole levels by tens of $\mathrm{meV}$ in these nanostructures, allowing us to restrict most of our discussions only to topmost (one or two) heavy hole levels. See Sections IIIA and IV.

Consider first the minimal model in which there is only one electron level and one hole level in the quantum dot. This is a reasonable assumption since the corresponding exciton is well isolated from the higher states. In a dot charged with one electron, there are two ground states $e_{-}^{\dagger}|G\rangle$ and $e_{+}^{\dagger}|G\rangle$ which represent the spin-up and spindown states of the doped electron with respect to the $z$ direction. $|G\rangle$ denotes the ground state of the quantum dot in the absence of the electron. There are two trion states $e_{-}^{\dagger} e_{+}^{\dagger} h_{+}^{\dagger}|G\rangle$ and $e_{+}^{\dagger} e_{-}^{\dagger} h_{-}^{\dagger}|G\rangle$. In the basis of $e_{-}^{\dagger}|G\rangle, e_{+}^{\dagger}|G\rangle, e_{-}^{\dagger} e_{+}^{\dagger} h_{+}^{\dagger}|G\rangle$ and $e_{+}^{\dagger} e_{-}^{\dagger} h_{-}^{\dagger}|G\rangle$ the Hamiltonian including the effect of external magnetic field and light-matter interaction has the form

$$
H=\left[\begin{array}{cc}
\omega_{B} g_{z}^{e} \cos \theta & \omega_{B} g_{x}^{e} \sin \theta \\
\omega_{B} g_{x}^{e} \sin \theta & -\omega_{B} g_{z}^{e} \cos \theta \\
\Omega_{+} e^{-i \omega_{+} t} & 0 \\
0 & \Omega_{-} e^{-i \omega_{-} t}
\end{array}\right.
$$

where $\omega_{B}=\frac{1}{2} \mu_{B}|\vec{B}|$ and $\theta$ is the angle between external magnetic field and $z$-axis. $E_{T}$ is the excitation energy of the trion state at zero magnetic field. The Hamiltonian can be used to calculate the linear absorption spectra of trions in various magnetic field configurations. For the heavy hole, $g_{x}^{h}$ is negligible if the heavy hole-light hole mixing and the $k^{3}$ terms in the Luttinger Hamiltonian are neglected. The Hamiltonian in Eq. (4) is the same one used by Tischler et $a l^{20}$ to deduce the g tensors from the magneto-photoluminescence measurements.

It is clear from Eq. (44) that the two spin ground states are not coupled by the applied oscillating electric field unless there is a mixing magnetic field tilted away from the $z$-axis. We shall present first the simple case of the 
Voigt configuration $\theta=\pi / 2$ in which the magnetic field is in the quantum well plane with its direction designated as the $x$-axis. Generalization to arbitrary field direction (see Section IIIB) is straightforward. The Voigt case is worth special attention because it is the simplest case for experimental implementation and it gives the simplest illustration of the underlying physics for the control of the single qubit operation. In the case where only $\sigma_{+}$ polarized light is used and setting $g_{x}^{h}=0$, the trion state $e_{+}^{\dagger} e_{-}^{\dagger} h_{-}^{\dagger}|G\rangle$ is decoupled from the rest. The magnetic field in the $x$ direction produces a Zeeman splitting between the states $e_{ \pm x}^{\dagger}|G\rangle=(1 / \sqrt{2})\left(e_{-}^{\dagger} \pm e_{+}^{\dagger}\right)|G\rangle$. The states $e_{+x}^{\dagger}|G\rangle, e_{-x}^{\dagger}|G\rangle$ and $e_{-}^{\dagger} e_{+}^{\dagger} h_{+}^{\dagger}|G\rangle=e_{-x}^{\dagger} e_{+x}^{\dagger} h_{+}^{\dagger}|G\rangle$ identify a three level system. Consider now two phaselocked $\sigma_{+}$polarized lasers pulses which give rise to an off-diagonal matrix element of the interaction Hamiltonian in Eq. (4) of the form

$$
\Omega_{+}(t)=\Omega_{1}(t) e^{i\left(\omega_{+}-\omega_{1}\right) t-i \alpha}+\Omega_{2}(t) e^{i\left(\omega_{+}-\omega_{2}\right) t},
$$

where $\alpha$ is the relative phase between the two real Rabi energies $\Omega_{1}(t), \Omega_{2}(t)$. This form of the pulses can be obtained with pulse-shaping techniques. The frequencies $\omega_{1}, \omega_{2}$ are chosen to satisfy the Raman conditions,

$$
\omega_{1}+\omega_{B} g_{x}^{e}=\omega_{2}-\omega_{B} g_{x}^{e}=\omega_{+} \equiv E_{T}-\Delta
$$

where $\Delta$ is the common Raman detuning (see Fig. 1(a)). In the rotating frame defined by $e^{\mp i \omega_{B} g_{x}^{e} t} e_{ \pm x}^{\dagger}|G\rangle$ and $e^{-i\left(E_{T}-\Delta\right) t} e_{-x}^{\dagger} e_{+x}^{\dagger} h_{+}^{\dagger}|G\rangle$, the Hamiltonian becomes

$$
H=\frac{1}{\sqrt{2}}\left[\begin{array}{ccc}
0 & 0 & \Omega_{1}(t) e^{i \alpha}+\Omega_{2}(t) e^{2 i g_{x}^{e} \omega_{B} t} \\
0 & 0 & \Omega_{1}(t) e^{-2 i g_{x}^{e} \omega_{B} t+i \alpha}+\Omega_{2}(t) \\
\Omega_{1}(t) e^{-i \alpha}+\Omega_{2}(t) e^{-2 i g_{x}^{e} \omega_{B} t} & \Omega_{1}(t) e^{2 i g_{x}^{e} \omega_{B} t-i \alpha}+\Omega_{2}(t) & \sqrt{2} \Delta
\end{array}\right]
$$

When $\left|\Omega_{j}(t)\right| \ll g_{x}^{e} \omega_{B}$, the fast oscillating terms can be neglected. Then the Hamiltonian takes the form

$$
H_{r}=\left[\begin{array}{ccc}
0 & 0 & e^{i \alpha} \Omega_{\uparrow}(t) \\
0 & 0 & \Omega_{\downarrow}(t) \\
e^{-i \alpha} \Omega_{\uparrow}(t) & \Omega_{\downarrow}(t) & \Delta
\end{array}\right]
$$

where $\Omega_{\uparrow}=\Omega_{1} / \sqrt{2}, \Omega_{\downarrow}=\Omega_{2} / \sqrt{2}$. This constitutes a single $\Lambda$ system as shown in Fig. I(a). For typical Zeeman splittings of $1 \mathrm{meV}$ and simple Gaussian pulses, frequency selectivity requires a pulse temporal width much longer than $0.6 \mathrm{ps}(=\hbar / 1 \mathrm{meV})$.

\section{CONTROL OF SPIN DYNAMICS IN A CHARGED DOT}

Stimulated Raman adiabatic passage (STIRAP) 28 has been extensively used to perform population transfer between quantum states. ${ }^{29}$ It has also been used to create entangled states ${ }^{30}$ In contrast to the typical STIRAP population transfer scheme, we do not make assumptions on the initial state of the system. The transformation we are considering are general rotations, independent of the initial orientation of the spin. A procedure to perform general spin rotation via STIRAP was proposed recently 31 However, an extra auxiliary ground state was required in addition to the two ground states. It is unsuited to the case of a single charged quantum dot. This STIRAP method can be used in coupled QDs for single qubit operations and quantum gates ${ }^{32.33}$ In the following we will first show how to perform an arbitrary spin rotation in a single $\Lambda$ system without using any auxiliary
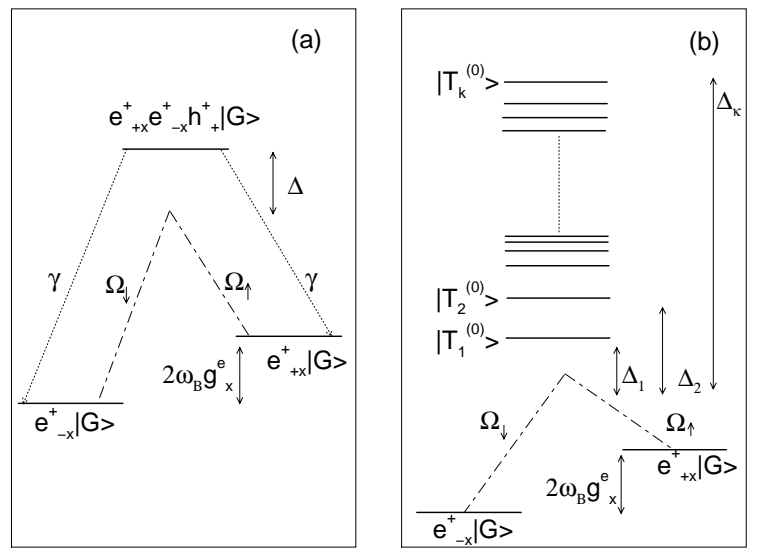

FIG. 1: Lambda systems in a QD. Only $\sigma+$ polarized light is used in the Voigt configuration. (a) Single trion model. (b) Multiple trion-level model. At low temperatures, the main decoherence mechanism is the spontaneous radiative decay of the trion state indicated by $\gamma$ in (a). $\Omega_{\uparrow}=\Omega_{1} / \sqrt{2}$, and $\Omega_{\downarrow}=$ $\Omega_{2} / \sqrt{2}$ are defined in Eq. [5] satisfying the Raman condition in Eq. 6]

level. We will discuss then the adiabatic condition and the effect of the decaying intermediate trion state. 


\section{A. General single-spin rotation}

The Hamiltonian of the single $\Lambda$ system in Eq. (8) may be diagonalized analytically by the substitutions,

$$
\begin{aligned}
\Omega_{\uparrow} & =\Xi \sin (2 \phi) \cos \beta, \\
\Omega_{\downarrow} & =\Xi \sin (2 \phi) \sin \beta, \\
\Delta & =2 \Xi \cos (2 \phi) .
\end{aligned}
$$

$\Xi(t)$ is the grand Rabi frequency,

$$
\Xi=\sqrt{\Omega_{\uparrow}^{2}+\Omega_{\downarrow}^{2}+\left(\frac{\Delta}{2}\right)^{2}}
$$

The angle $\phi(t)$ may be called roughly the tipping angle of the pseudo-magnetic field if the three states are regarded as pseudo-spin states. To make clear the physical meaning of $\beta$ below, it is convenient to make the two pulses, $\Omega_{\uparrow}(t)$ and $\Omega_{\downarrow}(t)$, with the same envelope shape. Then the angle $\beta=\arctan \left(\Omega_{\downarrow} / \Omega_{\uparrow}\right)$ is independent of time. In general, the pulse shape identity may be relaxed to the extent that the time independence of $\beta$ becomes a slowly varying one to satisfy the adiabatic condition to be considered next. The matrix of three columns of eigen-vectors,

$$
W(t)=\left[\begin{array}{ccc}
-e^{i \alpha} \sin \beta & -e^{i \alpha} \cos \beta \cos \phi & e^{i \alpha} \cos \beta \sin \phi \\
\cos \beta & -\sin \beta \cos \phi & \sin \beta \sin \phi \\
0 & \sin \phi & \cos \phi
\end{array}\right],
$$

leads to the diagonal form of the Hamiltonian at time $t$, $W^{\dagger}(t) H_{r}(t) W(t)$, with the eigenvalues along the matrix diagonal, respectively,

$$
\begin{aligned}
& \lambda_{1}(t)=0, \\
& \lambda_{2}(t)=-2 \Xi(t) \sin ^{2} \phi(t), \\
& \lambda_{3}(t)=2 \Xi(t) \cos ^{2} \phi(t) .
\end{aligned}
$$

The time-dependent eigenstates are used to form an adiabatic basis set. The effective Hamiltonian in this representation for the time-dependent Schrödinger equation is given by

$$
H_{a d}=W^{\dagger} H W-i W^{\dagger} \frac{d W}{d t} .
$$

If the second term on the right is neglible, the transformed Hamiltonian is diagonal, leading to a diagonal evolution operation $U_{a d}\left(t, t^{\prime}\right)$ with the terms $e^{-i \Lambda_{j}}, j=$ $1,2,3$, where $\Lambda_{i}=\int_{t^{\prime}}^{t} d t^{\prime \prime} \lambda_{i}\left(t^{\prime \prime}\right)$. Since $\Lambda_{1}=0$, the first eigenstate is time independent and completely decoupled from the other two states. The motion governed by the instantaneous eigenenergies is known as adiabatic. The condition for the adiabatic approximation is the slow time variation of $W$ which, from Eq. (11), depends on the rate of change of the tipping angle $\dot{\phi}(t)$ in comparing with the rate of the adiabatic motion given by the grand Rabi frequency which sets the magnitudes of the instantaneous eigenenergies,

$$
|\dot{\phi}(t)| \ll 2 \Xi(t)
$$

For the qubit operation, at $t=-\infty$ the state of the system is a linear combination in the subspace spanned by the eigenstates associated with $\lambda_{1}$ and $\lambda_{2}(-\infty)$. The time dependent Hamiltonian describing the optical pulses has a cyclic behavior, meaning that $H(t=\infty)=H(t=$ $-\infty)$. The idea of the adiabatic evolution is that, if the Hamiltonian varies slowly enough in time, the state of the system remains confined in the subspace spanned by the two eigenstates at all times. An arbitrary initial state in the spin ground state subspace, $[a, b]^{T}$ will acquire only a phase in the $\lambda_{2}$ component, transforming to $\left[a, e^{-i \Lambda_{2}} b\right]^{T}$. The evolution operator in the original rotating frame is given by

$$
\begin{aligned}
& U(+\infty,-\infty)=W(\infty) U_{a d} W^{\dagger}(-\infty) \\
& =\left[\begin{array}{ccc}
e^{-i \Lambda_{2} / 2} U_{2} & \vdots \\
& \vdots & 0 \\
\ldots \ldots \ldots \ldots \ldots \ldots & \ldots \ldots
\end{array}\right] \text {, }
\end{aligned}
$$

where

$$
U_{2}=e^{-\frac{i}{2} \Lambda_{2} \vec{\sigma} \cdot \vec{n}}
$$

is our final result for the rotation in the spin $1 / 2$ subspace through an angle $\Lambda_{2}$ about the unit vector $\vec{n}$ in the polar direction given by the declination and azimuthal angle, $(2 \beta, \alpha)$, or

$$
\begin{aligned}
& n_{1}=\cos \alpha \sin (2 \beta), \\
& n_{2}=-\sin \alpha \sin (2 \beta), \\
& n_{3}=\cos (2 \beta),
\end{aligned}
$$

where the Cartesian directions $(1,2,3)$ are along the unit vectors $(\hat{z},-\hat{y}, \hat{x})$. The polar direction is along the magnetic field. The effect of the spin precession due to the magnetic field is avoided by always working in the rotating frame introduced by Eq. (7).

The corrections due to the light-hole come in two forms. One is the light-hole mixing in the heavy-hole and electron singlet-pair trion state ${ }^{27}$ The spin-up electron is connected by the $\sigma_{+}$polarization to the $+3 / 2$ heavy-hole trion whose mixture with the $+1 / 2$ light-hole component is connected by the same polarized light to the spin down electron state. This induces an extra rotation of the order of $1 \%$ of $\Lambda_{2}$ about an axis normal to the growth axis, which is just a minor correction which can be included in the effect of the transverse magnetic field. The other correction is due to the light-hole trion whose effect is small if the detuning is less than $10 \mathrm{meV}^{33}$ and can be eliminated by pulse-shaping. 12,13

\section{B. Arbitrary magnetic field orientation}

Since the tilted magnetic field is essential to the complete set of single-qubit operations, it is important to 
study the dependence of the operation on the field orientation. The generalization to an arbitrary direction follows the same procedure as in Sections II and IIIA. Taking again only $\sigma+$ polarized light, we need to consider only the two spin ground states and one trion state made out of a spin-up $(+3 / 2)$ hole and two electrons in a singlet. We rewrite the reduced Hamiltonian from Eq. (4) in the appearance of a Hamiltonian with an effective $g^{e}$,

$$
H=\left[\begin{array}{ccc}
\omega_{B} g^{e} \cos \vartheta & \omega_{B} g^{e} \sin \vartheta & \Omega_{+}^{*}(t) e^{i \omega_{+} t} \\
\omega_{B} g^{e} \sin \vartheta & -\omega_{B} g^{e} \cos \vartheta & 0 \\
\Omega_{+}(t) e^{-i \omega_{+} t} & 0 & E_{T}+\omega_{B} g_{z}^{h} \cos \theta
\end{array}\right]
$$

where we have set $g_{x}^{h}$ to zero and defined the

$\theta$-dependent effective $g^{e}$ and the effective angle $\vartheta$ by

$$
\begin{aligned}
g^{e}(\theta) & =\sqrt{\left(g_{z}^{e} \cos \theta\right)^{2}+\left(g_{x}^{e} \sin \theta\right)^{2}} \\
\vartheta(\theta) & =\arctan \left(\frac{g_{x}^{e}}{g_{z}^{e}} \tan \theta\right) .
\end{aligned}
$$

By the unitary transformation

$$
U=\left[\begin{array}{ccc}
\cos \frac{\vartheta}{2} & \sin \frac{\vartheta}{2} & 0 \\
\sin \frac{\vartheta}{2} & -\cos \frac{\vartheta}{2} & 0 \\
0 & 0 & 1
\end{array}\right]
$$

the three basis states are transformed to the spin states along the field direction $| \pm B\rangle$, and the invariant trion $|T\rangle$. When the two pulses are chosen as in Eq. (5), the Hamiltonian $H_{r}$ in the new rotating frame is exactly of the same form as Eq. (8). The only changes are in the expressions for the Rabi energies and the detuning,

$$
\begin{aligned}
\Omega_{\uparrow} & =\Omega_{1} \cos \frac{\vartheta}{2}, \\
\Omega_{\downarrow} & =\Omega_{2} \sin \frac{\vartheta}{2}, \\
\Delta & =E_{T}+\omega_{B} g_{z}^{h} \cos \theta-\omega,
\end{aligned}
$$

The solution then follows exactly the procedure in Section IIA The resultant evolution yields the spin rotation as in Eq. (16). The Cartesian axes $(1,2,3)$ are along the unit vectors $(-\hat{y} \times \hat{B},-\hat{y}, \hat{B})$. As a check, note that if the magnetic field is parallel to the propagation axis of the light, then $\vartheta=0$ and we can realize only rotations about the $z$ axis. On the other hand, for a finite $\vartheta$ we can obtain a rotation about any axis by changing the control parameters $\alpha, \Omega_{1}, \Omega_{2}$ and $\Delta$.

\section{Suppression of trion decoherence}

It is physically reasonable that the use of the offresonance Raman processes should avoid the short optical decoherence time due to the rapid recombination of the exciton, since the the excited state is only virtually excited. The coherence of the spin dynamics is then governed by the much longer spin dephasing time. In a more quantitative study, we consider the eigenstates in Eq. (11). The first eigenstate $\left|\lambda_{1}\right\rangle$ has no component in the intermediate trion state, and the second eigenstate $\left|\lambda_{2}\right\rangle$ has only a small component in the intermediate state as long as $\Omega(t) / \Delta$ is small. As a result the intermediate state is only weakly populated during the Raman transition and its decoherence has a weak effect on the coherence of the spin rotation.

To substantiate this claim, we start with the master equation of the density matrix $\rho$,

$$
\frac{d \rho}{d t}=-i[H, \rho]-\frac{1}{2} \sum_{i}\left(L_{i}^{\dagger} L_{i} \rho+\rho L_{i}^{\dagger} L_{i}-2 L_{i} \rho L_{i}^{\dagger}\right)
$$

where $L_{i}$ are the Lindblad 34 operators. These operators have the form of projectors and describe the effect of the spontaneous radiative recombination of the trion state as shown in Fig. 1(a). The density matrix in the adiabatic representation is $\varrho=W^{\dagger} \rho W$ (note the slightly different symbol $\varrho$ used on the left) and satisfies the transformed equation

$$
\begin{aligned}
\frac{d \varrho}{d t}= & -i\left[H_{a d}, \varrho\right] \\
& -\frac{1}{2} \sum_{i}\left(M_{i}^{\dagger} M_{i} \varrho+\varrho M_{i}^{\dagger} M_{i}-2 M_{i} \varrho M_{i}^{\dagger}\right),
\end{aligned}
$$

where $M_{i}=W^{\dagger} L_{i} W$. The effect of the transformation on the Lindblad operators is considerably simplified if we assume that the spontaneous emission rates from the trion to the two spin ground states are the same, $\gamma$. By symmetry the results are independent of the rotations associated with the relative phase of the two pulses $\alpha$ and the rotation of the spin basis states to the magnetic field direction $\beta$. The total relaxation part is given by

$$
M_{\text {relax }}[\varrho]=-\gamma\left[\begin{array}{ccc}
0 & r_{1}^{\dagger} \sin \phi & r_{1}^{\dagger} \cos \phi \\
r_{1} \sin \phi & \left(r_{2}+r_{2}^{\dagger}\right) \sin \phi & r_{2}^{\dagger} \cos \phi+r_{3} \sin \phi \\
r_{1} \cos \phi & r_{2} \cos \phi+r_{3}^{\dagger} \sin \phi & \left(r_{3}+r_{3}^{\dagger}\right) \cos \phi
\end{array}\right]+\gamma r_{0}\left[\begin{array}{ccc}
1 & 0 & 0 \\
0 & \cos ^{2} \phi & -\sin \phi \cos \phi \\
0 & -\sin \phi \cos \phi & \sin ^{2} \phi
\end{array}\right]
$$

where $r_{j}=\varrho_{2, j} \sin \phi+\varrho_{3, j} \cos \phi, r_{j}^{\dagger}=\varrho_{j, 2} \sin \phi+\varrho_{j, 3} \cos \phi$, and $r_{0}=r_{2} \sin \phi+r_{3} \cos \phi$, for $j=1,2,3$. The origin of the decoherence in the Raman process may be exhibited by a simpler expression of $M_{\text {relax }}$ which is obtained by 
expansion in powers of the small quantity $\phi(t)$,

$$
M_{\text {relax }}[\varrho]=\gamma\left\{\left[\begin{array}{ccc}
\varrho_{33} & 0 & -\varrho_{13} \\
0 & \varrho_{33} & -\varrho_{23} \\
-\varrho_{31} & -\varrho_{32} & -2 \varrho_{33}
\end{array}\right]-\left[\begin{array}{ccc}
-\varrho_{23}-\varrho_{32} & \varrho_{13} & \varrho_{12} \\
\varrho_{31} & 0 & \varrho_{22}+2 \varrho_{33} \\
\varrho_{21} & \varrho_{22}+2 \varrho_{33} & \varrho_{23}+\varrho_{32}
\end{array}\right] \phi+O\left(\phi^{2}\right)\right\} .
$$

At the start of a qubit operation, the density matrix has the form

$$
\varrho(-\infty)=\left[\begin{array}{ccc}
\varrho_{11} & \varrho_{21} & 0 \\
\varrho_{21} & \varrho_{22} & 0 \\
0 & 0 & 0
\end{array}\right]
$$

If the adiabatic condition in Eq. (14) is satisfied, then $\varrho_{j 3} \forall j$ remain nearly zero (of first order in $\phi$ ) at all times. To first order in $\phi, M_{\text {relax }}[\varrho]$ is proportional only to $\varrho_{j 3}$ in the subspace spanned by $\left|\lambda_{1}\right\rangle$ and $\left|\lambda_{2}\right\rangle$. Hence, the relaxation terms in this subspace are of second order in $\phi$. This demonstrates a suppression of the optical decoherence effects within the adiabatic subspace.

A more quantitative measure of the qubit operation is the commonly-used fidelity which is an overlap of the physical operation versus the ideal. We follow the averaging over all possible initial states in the Hilbert space as was done in Refs. 12 and 13 . To compute the fidelity, we have performed a numerical simulation on the adiabatic spin rotation using the quantum trajectory method, 35 which is equivalent to solving the master equation with the relaxation terms in Eq. (25). We take the common shape of the pulses to be Gaussian, $\propto \exp -(t / \tau)^{2}$. The lifetime of the trion due to spontaneous emission (Fig. (1) is taken to be 60 ps. Other forms of dephasing, such as that induced by the electron-phonon interaction, are experimentally found to be negligible in the fluctuation quantum dots ${ }^{36}$ We simulate the operation of a $\pi$ rotation in the spin space. For a Rabi energy $\Omega_{0}=1 \mathrm{meV}$ and a detuning $\Delta=5 \mathrm{meV}$ we find an appropriate pulse duration given by $\tau=8.74 \mathrm{ps}$. The resultant fidelity of this operation is $F=0.991$. If the detuning is increased to $\Delta=10 \mathrm{meV}$, we find that the adiabaticity condition is better satisfied and the operation is more robust against spontaneous emission. The fidelity in this case increases to $F=0.995$. The price is a longer pulse duration, $\tau=16.74 \mathrm{ps}$ for the $\pi$ rotation. This demonstrates numerically that the decoherence of the intermediate trion state can be suppressed using an adiabatic control. Once the effect of spontaneous emission has been reduced, the spin-flip decoherence is the remaining limiting mechanism for the coherence of the qubit. This time has been found to be of the order of hundreds of nanosecond,, 22 and, therefore, we can afford to use rather long pulses for the control.

\section{MULTIPLE TRION LEVELS}

In this section we consider (i) what happens if more than one electron or hole levels are localized in the dot, and (ii) how to extend the theory from discrete trion levels to a continuum. In mono-layer fluctuation QDs and some cases of self-assembled dots, these continuum states are provided by delocalized excitons in the quantum well. We shall confine ourselves to the case of higher electronsinglet heavy-hole trion levels. There are light-hole effects which can be shown to be small as in Section IIIA. There are two-electron spin singlets and triplets. None of these are important if the detuning from the lowest single trion is small.

\section{A. Multiple Lambda system}

We assume that the initial state is still restricted to a linear combination of the spin ground states, $e_{1-}^{\dagger}|G\rangle$ and $e_{1+}^{\dagger}|G\rangle$. In the presence of many electron and hole levels in the dot, the effect of Coulomb interaction is to renormalize the trion energies and the oscillator strength of the optical transitions. Consider again the Voigt configuration. We have two $\sigma_{+}$laser pulses satisfying the two photon coherence configuration: $\omega_{1}+\omega_{B} g_{x}^{e}=\omega_{2}-\omega_{B} g_{x}^{e}=E_{T, 1}-\Delta_{1}$ where $E_{T, 1}$ is the lowest trion eigenstate energy including the effects of the Coulomb interaction. In this case, there are many possible trion states $T_{1}^{(0)} \cdots T_{k}^{(0)}$ resulting from the many confined levels, and one ends up with a multiple $\Lambda$ system, as depicted in Fig. 1(b). The general theory developed in section IIII can be extended to treat the multiple $\Lambda$ system ${ }^{37}$ To illustrate the method we consider the case where the two pulses are identical, i.e., $\Omega_{1}(t)=\Omega_{2}(t)$, and $\alpha=0$. This particular choice corresponds to a rotation about the $y$ axis. Let us change to the rotating basis, $| \pm\rangle \equiv\left(e^{-i \omega_{B} g_{x}^{e} t} e_{+x}^{\dagger}|G\rangle \pm e^{i \omega_{B} g_{x}^{e} t} e_{-x}^{\dagger}|G\rangle\right) / \sqrt{2}$. The trion states are in the rotating frame where $\left|T_{i}\right\rangle=e^{-i \omega t}\left|T_{i}^{(0)}\right\rangle$. The Hamiltonian in the basis $|-\rangle,|+\rangle,\left|T_{1}\right\rangle, \cdots,\left|T_{k}\right\rangle$ becomes

$$
H=\left[\begin{array}{ccccc}
0 & 0 & 0 & 0 & 0 \\
0 & 0 & \Omega_{1}(t) & \cdots & \Omega_{k}(t) \\
0 & \Omega_{1}(t) & \Delta_{1} & \cdots & 0 \\
\vdots & \vdots & \vdots & \ddots & \vdots \\
0 & \Omega_{k}(t) & 0 & \cdots & \Delta_{k}
\end{array}\right]
$$

where $\Omega_{k}=\beta_{k} \Omega_{1}(t) / \sqrt{2}$, and $\beta_{k}$ is the oscillator strength of the optical transition. The first eigenvalue is zero 
$\lambda_{1}(t)=0$. The second eigenvalue $\lambda_{2}(t)$ can be calculated exactly. However, it often suffices to work in the second order perturbation theory in which the analytic expression is

$$
\lambda_{2}(t)=-\sum_{i} \frac{\left|\Omega_{i}(t)\right|^{2}}{\Delta_{i}} .
$$

The corresponding eigenstates are

$$
\begin{aligned}
\left|\lambda_{1}(t)\right\rangle & =|-\rangle \\
\left|\lambda_{2}(t)\right\rangle & =|+\rangle-\sum_{i=1}^{k}\left|T_{i}\right\rangle \frac{\Omega_{i}(t)}{\Delta_{i}} \\
\left|\lambda_{i+2}(t)\right\rangle & =\left|T_{i}\right\rangle+|+\rangle \frac{\Omega_{i}(t)}{\Delta_{i}}
\end{aligned}
$$

The adiabatic condition can be expressed as $\left|\left\langle\lambda_{i}(t)\left|\frac{d}{d t}\right| \lambda_{2}(t)\right\rangle\right| \ll\left|\lambda_{i}(t)-\lambda_{2}(t)\right|$, for all $i>2$. The most stringent condition is of course for the lowest trion state which gives

$$
\frac{\dot{\Omega}_{1}(t)}{\Delta_{1}} \ll \Delta_{1} .
$$

When this condition is fulfilled spin rotation can be achieved via multiple intermediate trion states. The coherence of the rotation is again preserved by the virtual excitation of intermediate states.

\section{B. Continuum $\Lambda$ system}

STIRAP via continuum has been proposed for population transfer in atomic physics ${ }^{29.38}$ Several approaches have been proposed to avoid leakage and decoherence. 39 We show here how the adiabatic manipulation of a single spin can be realized in principle in presence of a continuum. The key for avoiding leakage and decoherence is again an excitation below the continuum edge. The continuum is thus only virtually excited and the coherence of the spin rotation is preserved. The treatment parallels that of the multiple $\Lambda$ system case in section IVA The eigenenergy of $\left|\lambda_{1}(t)\right\rangle=|-\rangle$ is again $\lambda_{1}(t)=0$. By means of Fano's method ${ }^{40}$ the eigenenergy of the other discrete state $\lambda_{2}(t)$ can be determined by the integral equation,

$$
\lambda_{2}(t)=\int d \epsilon g(\epsilon) \frac{|\Omega(\epsilon)|^{2}}{\left(\lambda_{2}(t)-\Delta_{\epsilon}\right)},
$$

where we have replaced the summation over the discrete $k$ by the integral over the energy with the density of states $g(\epsilon)$. At $t=-\infty$ the state $\left|\lambda_{2}(-\infty)\right\rangle=|+\rangle$. The eigenvector of the new discrete and continuum states $\left|\lambda_{\epsilon}(t)\right\rangle$ can be solved analytically (not shown here). The adiabatic condition then can be expressed as

$$
\left|\left\langle\lambda_{\epsilon}(t)\left|\frac{d}{d t}\right| \lambda_{2}(t)\right\rangle\right| \ll \Delta_{\epsilon} .
$$

The Fano approach thus allows us to obtain an analytical but complicated expression for this condition valid to all orders in $\Omega / \Delta$. However, a more stringent condition can be obtained by expanding Eq. (35) to second order in $\Omega / \Delta$ as is done for the multiple $\Lambda$ case. It can be shown that it is sufficient to require $\dot{\Omega}_{\epsilon=0}(t) / \Delta \ll \Delta$ where $\Delta$ is the detuning to the continuum edge to fulfill the adiabatic condition, which is analogous to Eq. (33). When this condition is fulfilled it is possible to perform an adiabatic Raman transition coherently via the continuum of intermediate states.

\section{CONCLUSIONS}

We have presented a theory for arbitrary rotations of the spin of a single electron in a quantum dot via Raman transitions in the adiabatic limit. Charged exciton states, or trions, play the role of the upper level in an effective lambda system. An arbitrary spin rotation may be performed by tailoring the relative phase and the relative intensities of two laser pulses as well as choosing the polarization of the light and the orientation of a static magnetic field. The explicit relations between the parameters of the laser pulses and the angle and the axis of the spin rotation are given. We investigate how the intermediate state decoherence is suppressed when the operations are performed in the adiabatic regime. We derive the adiabatic condition in lambda systems where additional discrete levels or a continuum of states are present. We show the calculations for a representative case (the monolayer fluctuation quantum dots) with values of the oscillator strengths and the characteristic energy separation taken from the experiments. We emphasize that our scheme works independently of the confinement properties of the dots, as long as the structure of the trion levels can be represented by the one in Fig. 1a or Fig. 1b. In principle, quantum dots could be engineered to optimize the fidelity of the operations with this particular control scheme. The theory developed here provides a useful blueprint for the realization of single qubit operations in spin-based quantum information processing.

\section{Acknowledgments}

This work was supported by ARDA/ARO-DAAD1901-1-0478, NSF DMR-0099572, and DARPA SpinS program. We thank Sophia Economou and Lucasz Cywinski for careful readings of the draft. 
1 Y. Yafet in New developments in semiconductors. Edited by P. R. Wallace, R. Harris and M. J. Zuckermann. (Noordhoff, Leyden 1973).

2 D. G. Thomas and J. J. Hopfield, Phys. Rev. 175, 1021 (1968).

${ }^{3}$ P. Hu, S. Geschwind, and T. M. Jedju, Phys. Rev. Lett. 37, 1357 (1976).

${ }^{4} \mathrm{~S}$. Geschwind and R. Romestain in Light Scattering in Solids IV. Edited by M. Cardona and G. Güntherodt. (Springer-Verlag, Berlin 1984).

5 A. Imamoglu et al., Phys. Rev. Lett. 83, 4204 (1999).

6 D. Jonathan, M.B. Plenio and P.L. Knight, Phys. Rev. A 62, 042307 (2000).

7 D.J. Wineland et al., arXiv:quant-ph/0212079 v2 (2003).

8 R. Zadoyan, D. Kohen, D.A. Lidar, and V.A. Apkarian, Chem. Phys. 266, 323 (2001).

9 P.R. Hammer, A. V. Turukhin, M. S. Shahriar, and J. A. Musser, Opt. Lett. 26, 361 (2001).

10 J.A. Gupta, R. Knobel, N. Samarth, D.D. Awschalom, Science 292, 2459 (2002).

11 J. Bao A.V. Bragas, J.K. Furdyna and R. Merlin, NatureMaterials 2, 175-179 (2003).

12 P. Chen, C. Piermarocchi, and L. J. Sham, Phys. Rev. Lett. 87, 067401 (2001).

13 C. Piermarocchi, P. Chen, Y.S. Dale, and L. J. Sham, Phys. Rev. B 65, 075307 (2002).

14 T. H. Stievater et al., Phys. Rev. Lett. 87133603 (2001).

15 H. Kamada et al., Phys. Rev. Lett. 87246401 (2001).

16 H. Htoon et al., Phys. Rev. Lett. 88087401 (2002).

17 A. Wojs and P. Hawrylak, Phys. Rev. B 51, 10880 (1995).

18 L. Landin et al., Science 280, 262 (1998).

19 A. Hartmann et al., Phys. Rev. Lett. 84, 5648 (2000).

20 J. G. Tischler, A. S. Bracker, D. Gammon and D. Park, Phys. Rev. B 66, 081310(R) (2002).

21 D. Loss and D. P. Di Vincenzo, Phys. Rev. A 57, 120 (1998).

22 J. A. Gupta, D. D. Awschalom, X. Peng, and A. P. Alivisatos, Phys. Rev. B 59, R 10421 (1999).
23 C. Piermarocchi, P. C. Chen, L. J. Sham, and D. G. Steel, Phys. Rev. Lett 89167402 (2002).

24 D. Bacon, J. Kempe, D.A. Lidar and K.B. Whaley, Phys. Rev. Lett. 85, 1758 (2000); D. P. Di Vincenzo, D. Bacon, J. Kempe, G. Burkard, K. B. Whaley, Nature 408, 339 (2000).

25 E. Pazy et al., Europhys. Lett. 62, 175 (2003).

${ }^{26}$ D. Gammon et al., Science 273, 87 (1996); M. W. Bertz et al. Solid State Commun. 8643 (1993).

27 S.-R. Eric Yang and L.J. Sham, Phys. Rev. Lett. 58, 2598 (1987).

${ }^{28}$ For review see K. Bergmann, H. Theuer, and B. W. Shore, Rev. Mod. Phys. 70, 1003 (1998); N. V. Vitanov, T. Halfmann, B. W. Shore, and K. Bergmann, Annu. Rev. Phys. Chem. 52, 763 (2001).

29 N. V. Vitanov and S. Stenholm, Phys. Rev. A 55, 648 (1997).

30 R. G. Unanyan, B. W. Shore, and K. Bergmann, Phys. Rev. A 63, 043405 (2001).

31 Z. Kis and F. Renzoni, Phys. Rev. A 65, 032318 (2002).

32 F. Troiani, E. Molinari and U. Hohenester, Phys. Rev Lett. 90, 206802 (2003).

33 T. Calarco, A. Datta, P. Fedichev, E. Pazy, and P. Zoller, Phys. Rev. A 68, 012310 (2003).

34 G. Lindblad, Commun. Math. Phys. 48, 119 (1976).

35 H. Carmichael, An open system approach to quantum optics, (Springer-Verlag, Berlin 1993).

36 N.H. Bonadeo et al., Phys. Rev. Lett. 81, 2759 (1998).

37 N. V. Vitanov and S. Stenholm, Phys. Rev. A 60, 3820 (1999).

38 L. P. Yatsenko, R. G. Unanyan, K. Bergmann, T. Halfmann, and B. W. Shore, Opt. Commun. 135, 406 (1997).

39 R. G. Unanyan, N. V. Vitanov, B. W. Shore, K. Bergmann, Phys. Rev. A 61043408 (2000); R. G. Unanyan, N. V. Vitanov, and S. Stenholm, Phys. Rev. A 57462 (1998).

40 U. Fano, Phys. Rev. 124, 1866 (1961). 